Case Report

\title{
Acquired Aorto-Right Ventricular Fistula following Transcatheter Aortic Valve Replacement
}

\author{
Muhammad Tariq Shakoor, ${ }^{1}$ Ashequl M. Islam, ${ }^{2}$ and Samia Ayub $^{3}$ \\ ${ }^{1}$ Baystate Medical Center, Tufts University School of Medicine, Springfield, MA 01199, USA \\ ${ }^{2}$ Division of Cardiology, Baystate Medical Center, Tufts University School of Medicine, Springfield, MA 01199, USA \\ ${ }^{3}$ Department of Pulmonary and Critical Care, University of Arkansas for Medical Sciences, Little Rock, AR 72205, USA
}

Correspondence should be addressed to Muhammad Tariq Shakoor; mts2703@hotmail.com

Received 13 December 2014; Revised 18 March 2015; Accepted 20 March 2015

Academic Editor: Yoshiro Naito

Copyright (C) 2015 Muhammad Tariq Shakoor et al. This is an open access article distributed under the Creative Commons Attribution License, which permits unrestricted use, distribution, and reproduction in any medium, provided the original work is properly cited.

\begin{abstract}
Transcatheter aortic valve replacement (TAVR) techniques are rapidly evolving, and results of published trials suggest that TAVR is emerging as the standard of care in certain patient subsets and a viable alternative to surgery in others. As TAVR is a relatively new procedure and continues to gain its acceptance, rare procedural complications will continue to appear. Our case is about an 89-year-old male with extensive past medical history who presented with progressive exertional dyspnea and angina secondary to severe aortic stenosis. Patient got TAVR and his postoperative course was complicated by complete heart block, aorto-RV fistula, and ventricular septal defect (VSD) formation as a complication of TAVR. To the best of our knowledge, this is the third reported case of aorto-RV fistula following TAVR as a procedural complication but the first one to show three complications all together in one patient.
\end{abstract}

\section{Introduction}

Aortic valve replacement is the mainstay treatment for symptomatic aortic stenosis (AS). In properly selected patients, surgery offers substantial improvement in symptoms and life expectancy. However, aortic valve surgery has significant risk in patients with multiple comorbidities, and in a small fraction of patients it can be of "extreme" risk. Catheterbased techniques for aortic valve implantation provide an alternative method for treating AS patients with unacceptably high estimated surgical risks [1]. Transcatheter aortic valve replacement (TAVR) techniques are rapidly evolving, and results of published trials suggest that TAVR is emerging as the standard of care in certain patient subsets and a viable alternative to surgery in others. As TAVR is a relatively new procedure and continues to gain its acceptance, rare procedural complications will continue to appear [2]. Aortoright ventricular (aorto-RV) fistula has been reported as a complication of aortic valve replacement surgery but to the best of our knowledge there are only a couple of case reports with TAVR [3]. We report a case with complete heart block, aorto-RV fistula, and ventricular septal defect (VSD) formation as a complication of TAVR.

\section{Case Presentation}

An 89-year-old male with a prior history of laryngeal cancer status after surgical resection and radiation therapy with residual dysphagia, coronary artery disease status after coronary artery bypass grafting in 1992, severe aortic stenosis status after balloon valvuloplasty in 2013, pulmonary fibrosis, COPD on 2-liter continuous home $\mathrm{O}_{2}$ therapy, and TIA presented in our clinic with several years of progressive dyspnea and angina with exertion. He was admitted in the hospital at least five times over the past one year with chest pain and dyspnea. Diagnostic cardiac catheterization was done showing three out of four grafts being patent (SVGRPLV completely occluded). Last year, he underwent balloon aortic valvuloplasty with a $22 \mathrm{~mm}$ balloon for severe aortic stenosis and the patient reported partial relief of symptoms for approximately six months after the procedure but now the symptoms are progressively getting worse. Transthoracic 


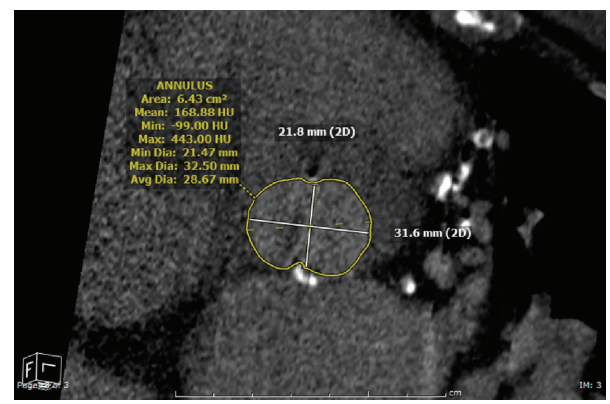

FIGURE 1: CT determined cross-sectional aortic annular dimensions.

echocardiogram (TTE) showed concentric left ventricular hypertrophy, left ventricular ejection fraction of $60 \%$, right ventricle mildly dilated with preserved systolic function, pulmonary artery systolic pressure of $34 \mathrm{mmHg}$, heavily calcified aortic valve annulus, mean pressure gradient of $68 \mathrm{mmHg}$, velocity of $517 \mathrm{~cm} / \mathrm{sec}$, and aortic valve area of $0.62 \mathrm{~cm}^{2}$. Cardiothoracic surgery team evaluated him for aortic valve replacement but the surgeon felt that patient is at very high risk for open-heart surgery. Per our TAVR team, his aortic valve annulus was too big (anteroposterior diameter $2.6 \mathrm{~cm}$ and mediolateral diameter of $2.8 \mathrm{~cm}$ per transesophageal echocardiogram, Figure 1) for commercially available Core Valve (Medtronic, USA). Ultimately, our TAVR team used a $29 \mathrm{~mm}$ Edward Sapien valve via transfemoral access. The procedure was complicated by complete heart block requiring temporary pacemaker backup. A permanent pacemaker was placed after 24 hours. Repeat TTE showed moderately dilated right ventricle with moderately reduced systolic function, pulmonary artery systolic pressure of $40 \mathrm{mmHg}$, Sapien prosthesis in aortic position with mild perivalvular leak and mean gradient of $9 \mathrm{mmHg}$, moderate sized continuous (systolic and diastolic phases) shunt which communicates with the aortic root around the prosthesis into the RV (aorto-RV shunt), and pulmonary flow (Qp) to systemic flow (Qs) ratio of 1.39 . The patient was managed conservatively with close follow-up. At 30-day follow-up, the patient remained moderately to severely dyspneic. Patient was offered percutaneous closure of the aorto-RV fistula using devices like septal occluder (Amplatzer device) but patient refused it. His repeat TTE showed worsening of aortoRV fistula and it also showed new small membranous VSD (see Video 1 in the Supplementary Material available online at http://dx.doi.org/10.1155/2015/608539), which was not visible on the first postprocedural TTE. His condition progressively got worse. He changed his code status to comfort measures only and passed away in a couple of days.

\section{Discussion}

Complications of TAVR include low cardiac output during and following deployment, annular rupture, vascular complications, myocardial injury, ventricular septal defect, heart block, paravalvular aortic regurgitation, renal failure, and stroke. 2 common procedural complications associated with TAVR have been well studied, whereas knowledge of

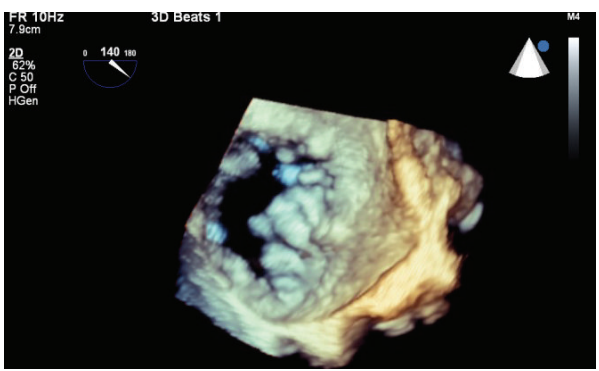

FIGURE 2: TEE three-dimensional view of aortic valve showing heavy calcification.

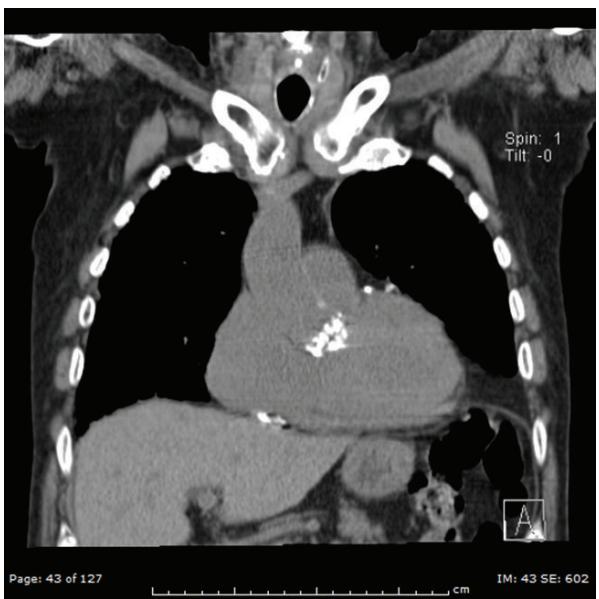

FIGURE 3: Coronal section of CT chest showing heavily calcified aortic valve.

rare procedural complications and subsequent management rely heavily on experience from case reports. Aortocardiac fistulas are relatively rare. Often, they are a complication of physical/surgical trauma or infective endocarditis [4]. A $2 \times 2 \mathrm{~cm}$ contact surface between the aorta above the right coronary cusp and the right ventricular outflow tract is the target area for an aorto-right ventricular fistula [4]. In our case the local tissue was heavily calcified and mildly aneurysmal right coronary sinus was seen on aortogram (Figures 2 and 3). So complications may have resulted from displacement of the calcified tissue causing local trauma. The clinical presentation of aorto-RV fistula depends on the size of the shunt. The natural history of aorto-RV fistula after TAVR is unknown but natural history of traumatic aorto-RV fistula was reported by Samuels et al. [4]. In their series of 40 patients, symptoms of heart failure developed at variable intervals and definitive surgical repair was performed in 38 of 40 patients. The mean interval between the time of injury and definitive repair was 1.5 years but in this series 35\% of patients had concomitant aortic valve regurgitation; thus it is not clear whether surgery was needed as a result of the aortic valve regurgitation or the large left to right shunt. Nevertheless, spontaneous closure of aorto-RV fistula has not been reported, so careful follow-up of all patients with aorto-RV fistula is prudent. With advances in interventional cardiology, it is now possible to close the aorto-RV fistula 
percutaneously using devices like septal occluder with threedimensional TEE guidance in patients who are poor surgical candidates. In our patient 30 days follow up TTE showed a new VSD, which may have resulted from the extension of aorto-RV fistula or perhaps it was missed in first TTE (Video 1).

\section{Conclusion}

To the best of our knowledge, this is the third reported case of aorto-RV fistula following TAVR as a procedural complication. The mechanism responsible for acquired aorto$\mathrm{RV}$ fistula is unknown but it can be related to trauma from displacement of calcified or abnormal tissue or the depth of prosthesis implantation. Conservative management is appropriate in patients without evidence of worsening symptoms and close follow-up is recommended with serial TTE, paying particular attention to right ventricle dimensions and pulmonary artery pressure. If symptoms are getting worse, percutaneous closure of the defect can be considered in this surgically high-risk population.

\section{Conflict of Interests}

Muhammad Tariq Shakoor, being the first author of the paper, solemnly declares that there is no conflict of interests among authors.

\section{References}

[1] J. G. Webb and D. A. Wood, "Current status of transcatheter aortic valve replacement," Journal of the American College of Cardiology, vol. 60, no. 6, pp. 483-492, 2012.

[2] M. B. Leon, N. Piazza, E. Nikolsky et al., "Standardized endpoint definitions for transcatheter aortic valve implantation clinical trials: a consensus report from the valve academic research consortium," Journal of the American College of Cardiology, vol. 57, no. 3, pp. 253-269, 2011.

[3] T. Pilgrim, B. Meier, and P. Wenaweser, "Aorto-right ventricular fistula after transfemoral aortic valve implantation," The Journal of Invasive Cardiology, vol. 22, no. 2, pp. E30-E31, 2010.

[4] L. E. Samuels, M. S. Kaufman, J. Rodriguez-Vega, R. J. Morris, and S. K. Brockman, "Diagnosis and management of traumatic aorto-right ventricular fistulas," Annals of Thoracic Surgery, vol. 65, no. 1, pp. 288-292, 1998. 


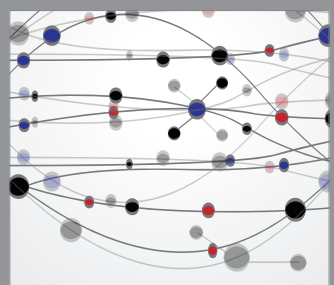

The Scientific World Journal
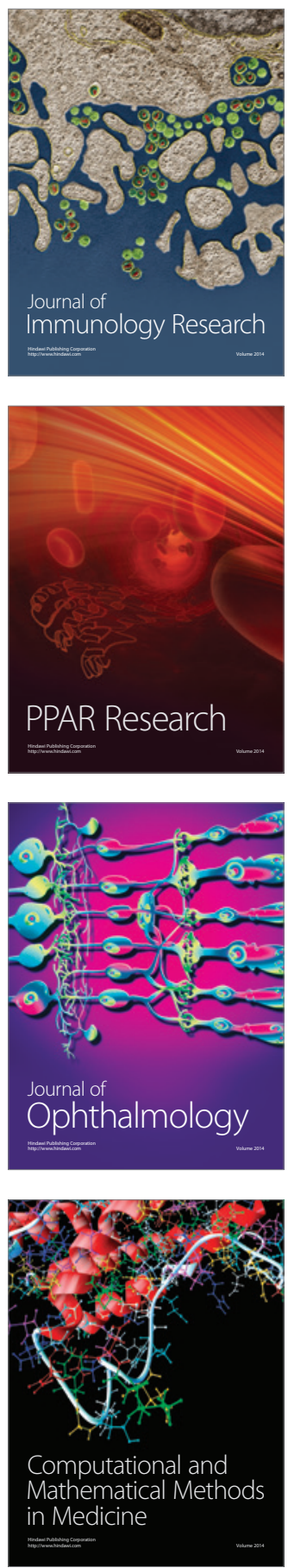

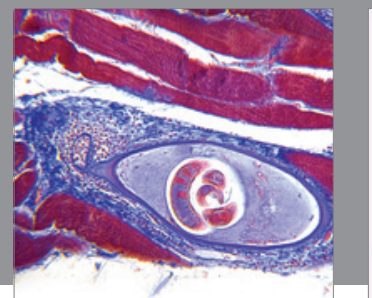

Gastroenterology

Research and Practice
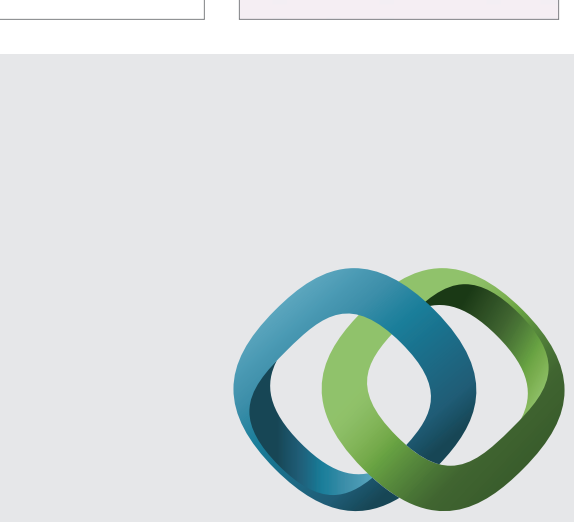

\section{Hindawi}

Submit your manuscripts at

http://www.hindawi.com
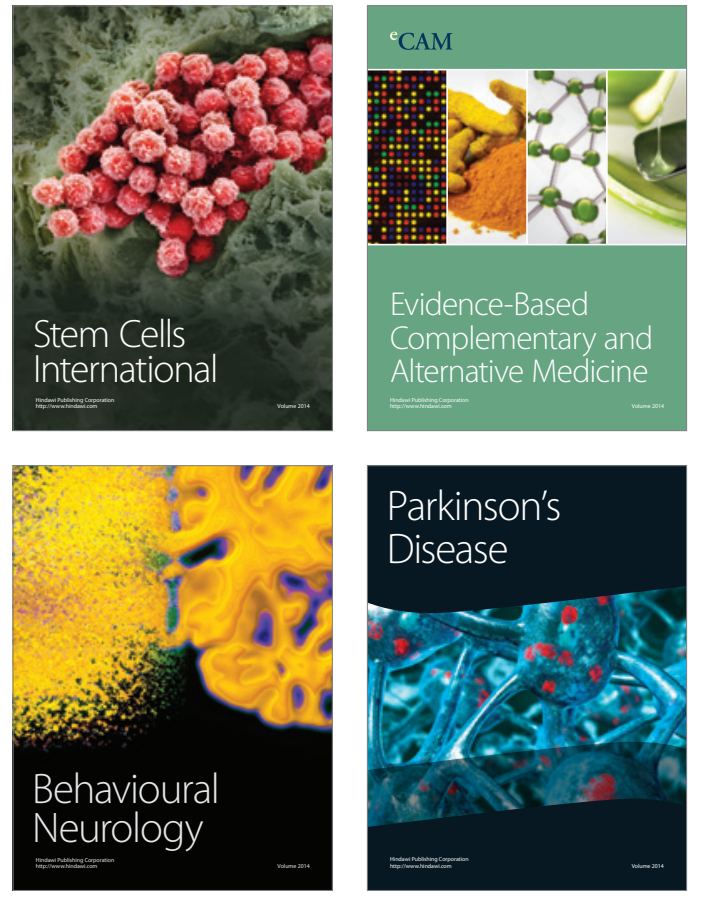
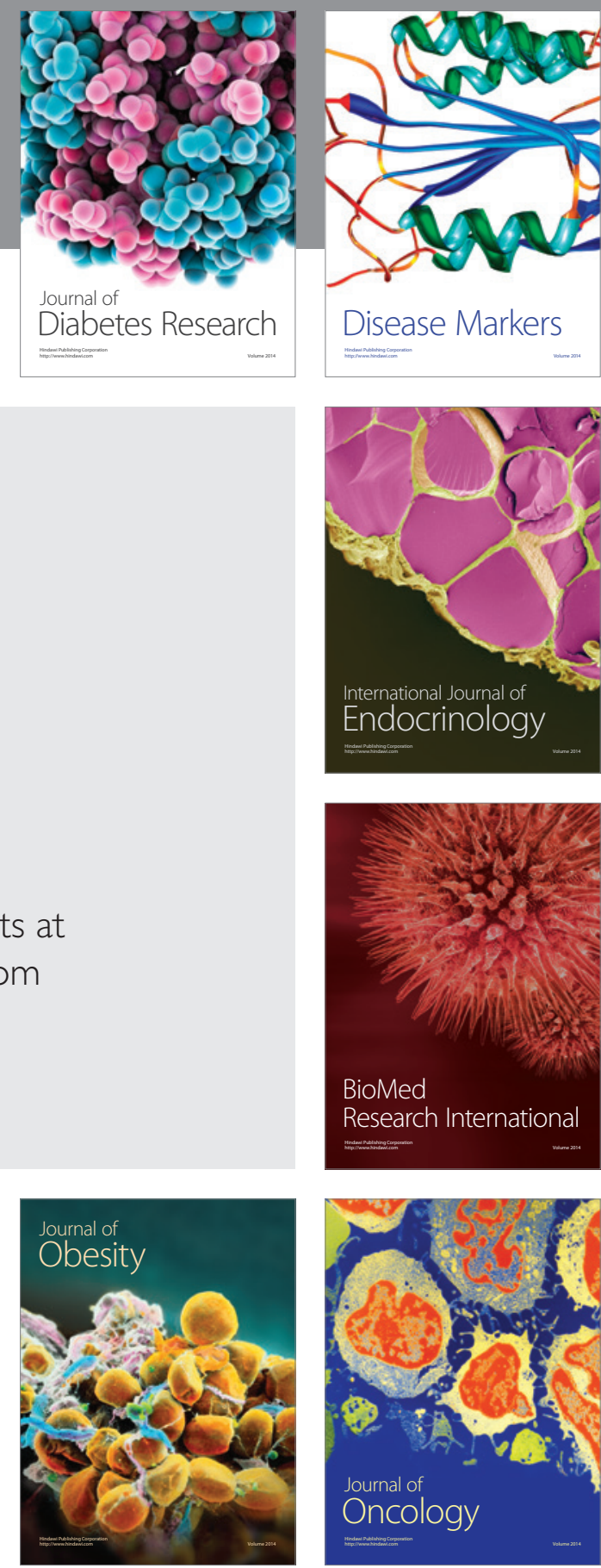

Disease Markers
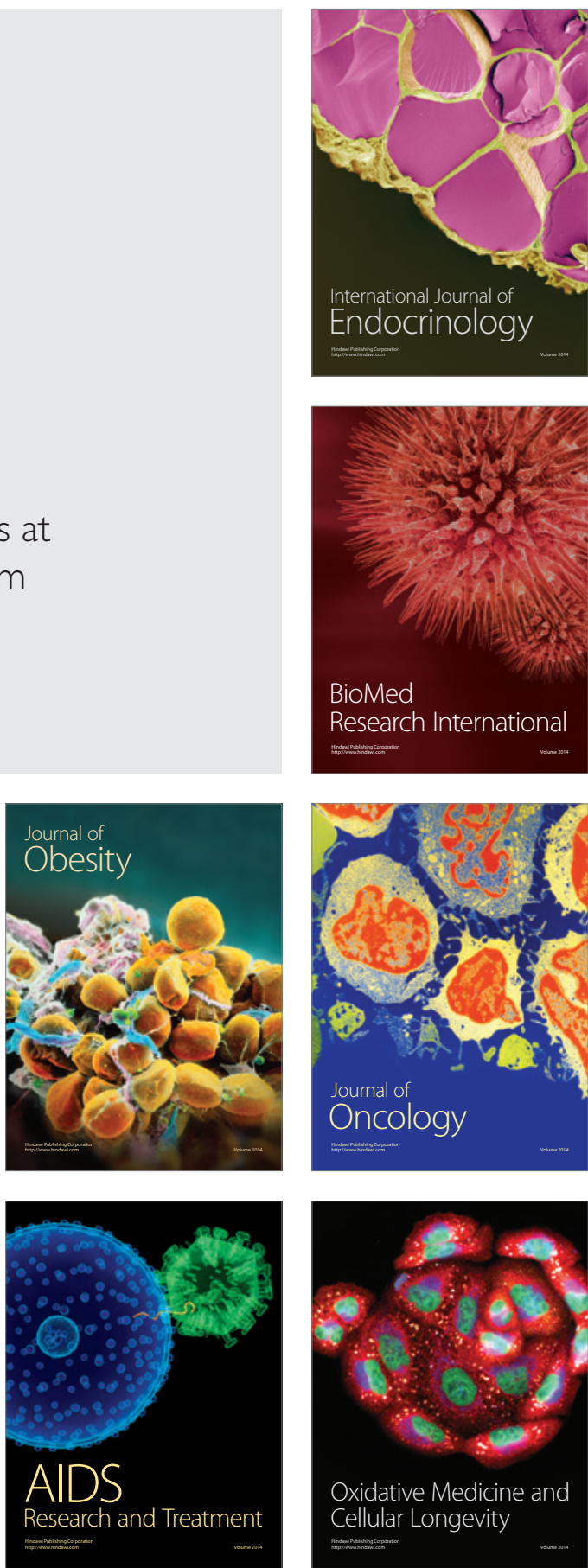